\title{
Stability of African swine fever virus on contaminated spray dried porcine plasma
}

\author{
Melina Fischer ${ }^{1}$, Jutta Pikalo², Martin Beer ${ }^{3}$, and Sandra Blome ${ }^{1}$ \\ ${ }^{1}$ Friedrich-Loeffler-Institute \\ ${ }^{2}$ Friedrich-Loeffler-Institute Federal Research Institute for Animal Health \\ ${ }^{3}$ Friedrich-Loeffler-Institut
}

January 17,2021

\begin{abstract}
African swine fever (ASF) is a viral disease that affects members of the Suidae family. The notifiable disease is considered a major threat to the pig industry, animal health, and food security worldwide. According to the European Food Safety Authority, ASF virus (ASFV) survival and transmission in feed and feed materials is a major research gap. Against this background, the objective of this study was to determine the survival of ASFV on re-contaminated spray dried porcine plasma (SDPP) when stored at two different temperatures. To this means, commercial SDPP granules were contaminated with high titers of ASFV in a worst-case re-contamination scenario. Three samples per time point and temperature condition were subjected to blind passaging on macrophage cultures and subsequent haemadsorption test to determine residual infectivity. In addition, viral genome was detected by real-time PCR. The results indicate that heavily re-contaminated SDPP stored at $4^{\circ} \mathrm{C}$ remains infectious for at least five weeks. In contrast, contaminated SDPP stored at room temperature displayed a distinct ASFV titer reduction after one week and complete inactivation after two weeks. In conclusion, the residual risk of ASFV transmission through re-contaminated SDPP is low, if SDPP is stored at room temperature for a period of at least two weeks before feeding.
\end{abstract}

\section{Introduction}

African swine fever (ASF) is considered a major threat to the pig industry, animal health, and food security worldwide (Guinat et al., 2016). The causative agent, ASF virus (ASFV) is a complex, enveloped DNA virus of the Asfarviridae family with high tenacity (Alonso et al., 2018). Given the stability of ASFV under a wide range of ambient conditions, farmers and other stakeholders are concerned about ASFV transmission via feed and feed ingredients, but unfortunately, the data basis is very limited. Against this background, the European Food Safety Authority (EFSA) defined research gaps regarding the potential for ASFV transmission through contaminated feed and feed materials (Alvarez et al., 2019). Of special interest could be feed with compounds of porcine origin, e.g. spray dried porcine plasma (SDPP). SDPP is extensively used in pig starter diets and consistently improves growth performance, and survival, especially under stressful conditions like pathogen challenge (Perez-Bosque et al., 2016). So far, there are very few studies on ASFV inactivation in re-contaminated porcine plasma. Kalmar et al. (2018) investigated a combination of physical and chemical processing conditions of liquid plasma, i.e. heat treatment $\left(48^{\circ} \mathrm{C}\right)$, alkaline conditions $(\mathrm{pH} 10.2)$, and addition of peroxide $\left(102.9 \mathrm{mM} \mathrm{H}_{2} \mathrm{O}_{2}\right)$. This treatment led to an ASFV titer reduction of $4.17 \log _{10} \mathrm{TCID}_{50} / \mathrm{ml}_{\mathrm{after}}$ $10 \mathrm{~min}$. Polo et al. (2019) reported a titer reduction of $4.62 \log _{10} \mathrm{TCID}_{50} / \mathrm{ml}$ after UV-C irradiation of liquid porcine plasma at a dose of $3000 \mathrm{~J} / \mathrm{l}$. The spray drying process itself with an inlet temperature of $200^{\circ} \mathrm{C}$ and a temperature of $80^{\circ} \mathrm{C}$ throughout substance led to an ASFV titer reduction of $4.11 \log _{10} \mathrm{TCID}_{50} / \mathrm{ml}$ (Blázquez et al., 2018). The above-mentioned studies addressed mainly contaminations that affected the raw materials, i.e. plasma originating from infected pigs. However, concerns were also raised with regard to possible re-contamination scenarios. 
The objective of the presented study was therefore to determine the stability of ASFV on re-contaminated SDPP after production when stored for different time periods $(0,7,14,21,28$ and 35 days) at two different temperatures $\left(4^{\circ} \mathrm{C}\right.$ and room temperature). For this purpose, commercial SDPP granules were contaminated with high titer ASFV ( $10^{6} 50 \%$ haemadsorbing doses per $\left.\mathrm{ml}\left[\mathrm{HAD}_{50} / \mathrm{ml}\right]\right)$. Successful contamination was demonstrated via real-time PCR analysis. Three samples per time point and temperature condition were subjected to a blind passage on peripheral blood mononuclear cell (PBMC) derived macrophages and to subsequent haemadsorption tests (HAT) to determine residual infectivity.

\section{Material and Methods}

\section{Sample preparation}

Spray dried porcine plasma (SDPP) from a commercial producer (APC, Villarrobledo, Spain) was used in this study. Seventy grams of SDPP (humidity max. 9.0\%) were contaminated with $10.5 \mathrm{ml}$ of an ASFV suspension with a titer of $10^{6} \mathrm{HAD}_{50} / \mathrm{ml}$ (ASFV strain "Armenia08"). The contamination procedure was performed in a zipper bag and divided into two steps $(5 \mathrm{ml}+5.5 \mathrm{ml}$ virus suspension), separated by a 15 min drying period, using an intranasal mucosal atomization device (MAD Nasal; Wolfe Tory Medical, Salt Lake City, USA) to nebulize the virus suspension. After additional $15 \mathrm{~min}$ drying and shaking at room temperature, contaminated SDPP was dispensed as $2 \mathrm{~g}$ aliquots in $50 \mathrm{ml}$ tubes (Sarstedt, Nümbrecht, Germany) and stored at $4^{\circ} \mathrm{C}$ or at room temperature. SDPP samples were taken as biological triplicates on days $0,7,14,21,28,35$ and stored at $-80^{\circ} \mathrm{C}$ until further analysis. As negative control ( $\mathrm{NC}$ ), triplicate samples of uncontaminated SDPP were stored immediately at $-80^{\circ} \mathrm{C}$ together with an aliquot of the original virus suspension $\left(\mathrm{VS} \mathrm{T}_{0}\right.$ ). Furthermore, $900 \mu \mathrm{l}$ of the virus suspension were also incubated at $4^{\circ} \mathrm{C}$ or at room temperature for 7 days or 35 days and subsequently stored at $-80^{\circ} \mathrm{C}$.

Prior to analysis, the $-80^{\circ} \mathrm{C}$ stored SDPP samples were resuspended thoroughly with $10 \mathrm{ml}$ sterile distilled water with an 1\% Antibiotic-Antimycotic mix (Gibco Antibiotic-Antimycotic 100x; Thermo Fisher Scientific, Schwerte, Germany), by shaking and vortexing. Two $\mathrm{ml}$ of the reconstituted plasma were stored at $-80^{\circ} \mathrm{C}$ for real-time PCR analysis to prove successful contamination. The remaining plasma was used as inoculum for a blind passage on peripheral blood mononuclear cell (PBMC) derived macrophages to determine whether any residual infectious ASFV could be detected.

\section{Isolation of macrophages, blind passage and haemadsorption test (HAT)}

For blind passage and haemadsorption test (HAT), isolation of PBMC derived macrophages was performed from EDTA-anticoagulated blood from healthy domestic pigs as previously described (Fischer et al., 2020). PBMCs were seeded into 6-well tissue culture plates (Primeria; Corning, Durham, USA) with a density of 1x $10^{7}$ cells/ml in Iscove's Modified Dulbecco's Medium with Ham's F-12 Nutrient Mix (Thermo Fisher Scientific, Schwerte, Germany) supplemented with 10\% fetal calf serum (FCS) and 1\% Antibiotic-Antimycotic mix (Thermo Fisher Scientific, Schwerte, Germany). Cells were cultured at $37 \mathrm{deg}$ C in a humidified atmosphere containing $5 \% \mathrm{CO}_{2}$ for 24 hours. Subsequently, medium was changed to remove non-adherent cells, adherent macrophages were rinsed with $1 \mathrm{ml}$ phosphate buffered saline (PBS) and afterwards wells were replenished with medium supplied with $2 \mathrm{ng} / \mathrm{ml}$ GM-CSF (granulocyte macrophage colony-stimulating factor; Biomol, Hamburg, Germany) to facilitate maturation of macrophages. PBMCs were incubated for 24 hours as described above. For blind passaging, medium was removed and replaced by $1 \mathrm{ml}$ of reconstituted plasma (inoculum). After a $2 \mathrm{~h}$ adsorption period at $37 \mathrm{degC}$ the inoculum was discarded and cultures were rinsed with $1 \mathrm{ml}$ PBS. Afterwards fresh medium without GM-CSF was added. Plates were incubated for $72 \mathrm{~h}$ and then stored at -80degC until real-time PCR analysis and processing in HAT.

For proof of infectious virus, PBMCs were seeded into 96-well microplates (Primeria; Corning, Durham, USA) with a density of $5 \times 10^{6}$ cells $/ \mathrm{ml}(100 \mu \mathrm{l}$ per well) and cultured as described above. Subsequently, 100 $\mu \mathrm{l}$ of each blind passage sample was used to inoculate four wells of a 96-well plate respectively (quadruplicate values). Furthermore, virus suspension samples were prepared for endpoint titration. To this means, 10-fold dilution series of these samples were inoculated in quadruplicate. After an $24 \mathrm{~h}$ incubation at $37^{\circ} \mathrm{C}, 20 \mu \mathrm{l}$ of a $1 \%$ homologous erythrocyte suspension in PBS were added to each well. For read-out, cultures were 
analyzed for haemadsorption reactions (formation of rosettes) over a period of 4 days. In a second-round HAT, virus positive blind passage samples and virus suspension samples were titrated in quadruplicate to obtain endpoint titers of biological replicates. Because of virus dilution and the number of replicate wells, the limit of detection is $1.75 \log _{10} 50 \%$ haemadsorbing doses per $\mathrm{ml}\left(\mathrm{HAD}_{50} / \mathrm{ml}\right)$. Titers were calculated using the Reed and Muench method (Reed \& Muench, 1938), and were expressed as $\log _{10} \mathrm{HAD}_{50} / \mathrm{ml}$. Graphical representation and calculations were performed using GraphPad Prism 8 (GraphPad Software Inc, San Diego, USA) and Excel version 2013 (Microsoft GmbH, Unterschleißheim, Germany), respectively.

\section{Nucleic acid extraction and real-time PCR}

Prior to real-time PCR analysis, viral nucleic acids from all samples (inoculum, blind passage, virus suspension) were extracted using the NucleoMag VET kit (Macherey-Nagel, Düren, Germany) on the automated KingFisher 96 flex platform (Thermo Fisher Scientific, Schwerte, Germany) according to the manufacturer's recommendations. Subsequently, nucleic acids were analyzed using a published real-time PCR (King et al., 2003) in combination with an internal control based on an EGFP detection system (Hoffmann et al., 2006) on a Biorad CFX real-time cycler (Bio-Rad Laboratories, Hercules, USA). For each real-time PCR, a quantification cycle $(\mathrm{Cq})$ value was determined according to the PCR cycle number at which the fluorescence of the reaction crosses a value that is statistically higher than the background which is determined by the Biorad CFX software. Using a dilution series of an in-house ASFV DNA standard, the genome copies in the respective samples were determined. For generation of the ASFV standard, DNA from an ASFV "Armenia08" PBMC culture supernatant was extracted using the QIAamp Viral RNA Mini Kit (Qiagen, Hilden, Germany) according to the manufacturer's recommendations. Subsequently, the DNA concentration was determined by spectrophotometry using a Nanodrop 2000c (Thermo Fisher Scientific, Schwerte, Germany) and the exact number of DNA molecules was calculated using an online tool (http://www.molbiol.edu.ru/eng/scripts/01 07.html).

\section{Results and Discussion}

Real-time PCR analysis of virus suspensions, contaminated SDPP and blind passages

The virus suspension used to contaminate SDPP contained high genome copy numbers $\left(5.66 \log _{10} / \mathrm{ml}\right.$, Figure 1). As a control, the original virus suspension was stored up to 35 days under the same conditions as the SDPP samples. Irrespective of the storage temperature $\left(4^{\circ} \mathrm{C}\right.$ or room temperature, respectively), ASFV genome copy numbers were constant over the entire observation period in the virus suspension (copy numbers between 5.66 and $5.39 \log _{10} / \mathrm{ml}$ ). However, copy numbers do not allow any conclusions on the amount of infectious virus.

In comparison, the contaminated SDPP, used as inoculum for the blind passage, contained lower genome copy numbers than the initial virus suspension due to dispersion on SDPP. Such a dispersion effect was also seen after contamination of field crops (Fischer et al., 2020). Overall ASFV genome was detectable in all samples, indicating a successful contamination of SDPP (Figure 1). Furthermore, genome copy numbers of SDPP did not display any distinct decline over time, irrespective of the storage temperature. In the un-treated plasma negative control (NC) no ASFV genome was detected (Table S1).

PCR analyses after blind passages of contaminated SDPP stored at $4^{\circ} \mathrm{C}$ revealed that these specimens were highly genome positive during the entire observation period (copy numbers between 7.32 and $6.52 \log _{10} / \mathrm{ml}$, Figure 2A). These values exceed the viral load of the initial virus suspension, indicating a considerable amplification of ASFV on macrophages during the blind passages. In contrast, only a single blind passage replicate of SDPP stored at room temperature for one week was PCR positive ( $4.88 \log _{10}$ genome copies $/ \mathrm{ml}$, Figure 2A and Table S2).

\section{Endpoint titers of virus suspension and blind passage}

In general, endpoint titers of the virus suspensions remained stable over a five-week storage period at $4^{\circ} \mathrm{C}$. At room temperature, virus titers declined from 6.0 to $4.13 \log _{10} \mathrm{HAD}_{50} / \mathrm{ml}$ over time (Table S3). Dee et al. (2018) also observed a comparable titer reduction (from 5.0 to $3.0 \log _{10} \mathrm{TCID}_{50}$ ) in a virus stock during 
30 days of storage mimicking transatlantic shipment conditions. Overall, the virus suspension remained infectious over the entire observation period, irrespective of the storage temperature.

Regarding blind passage samples of contaminated SDPP stored at $4^{\circ} \mathrm{C}$, virus titers remained constant over the entire study period (between 7.75 and $6.50 \log _{10} \mathrm{HAD}_{50} / \mathrm{ml}$, Figure 2B). Thus, contaminated SDPP stored at $4^{\circ} \mathrm{C}$ was infectious for at least five weeks. In contrast, blind passage samples of contaminated SDPP stored at room temperature displayed only residual ASFV infectivity (one out of three replicates HAT positive) and a distinct titer reduction after one week (Figure 2B). After two weeks, complete ASFV inactivation was observed. This finding is in accordance with the corresponding real-time PCR results (Figure 2A). In comparison to PEDV, which is fully inactivated on spray dried bovine plasma after three weeks at $4^{\circ} \mathrm{C}$ or after one week at room temperature (Pujols and Segalés, 2014), ASFV displayed a higher stability on SDPP.

When judging these results, the sensitivity of the HAT detection system should be taken into consideration. Compared with a bioassay, i.e. the inoculation of pigs, the HAT is less sensitive (Heuschele, 1967). However, this discrepancy in sensitivity should not be a concern in this case, since Blázquez et al. (2020) demonstrated that the minimum infectious dose in feed mixed with ASFV contaminated liquid porcine plasma was higher than $5.0 \log _{10} \mathrm{TCID}_{50} / \mathrm{pig}(50 \%$ tissue culture infectious dose per pig).

SDPP as a risk factor for ASFV transmission

It has been shown that the spray drying process itself has an inactivating effect on various viruses affecting pigs, like swine vesicular disease virus (SVD), pseudorabies virus (PRV), porcine reproductive and respiratory syndrome virus (PRRSV) or ASFV (Blázquez et al., 2018, Polo et al., 2005, Pujols and Segalés, 2014, Pujols et al., 2007). Hence, we investigated the re-contamination of SDPP after processing as well as the possibility of residual ASFV infectivity after an initial titer reduction during production. Thereby this study provides valuable data for evaluating the risk of ASFV transmission via contaminated SDPP. Based on the obtained results, we conclude that the residual risk of re-contamination of SDPP that is produced to European standards can be mitigated by room temperature storage for at least 14 days.

\section{Author Contributions}

Conceptualization, MF and SB; Data curation, MF; Funding acquisition, SB and MB; Investigation, MF and JP; Methodology, MF, and JP; Visualization, MF; Writing - original draft, MF and SB; Writing - review \& editing, JP and MB. All authors have read and agreed to the published version of the manuscript.

\section{Funding}

This study received funding through the FLI Internal ASF Research Network.

\section{Acknowledgments}

The authors would like to thank Ulrike Kleinert and Robin Brandt for their excellent technical assistance. Spray dried porcine plasma was kindly provided by APC Europe.

\section{Conflict of interest statement}

The authors declare no conflict of interest.

\section{Data availability statement}

The data that support the findings of this study are available from the corresponding author upon reasonable request.

\section{References}

Alonso, C., M. Borca, L. Dixon, Y. Revilla, F. Rodriguez, J. M. Escribano and C. Ictv Report, 2018: ICTV Virus Taxonomy Profile: Asfarviridae. J Gen Virol, 99, 613-614. 
Alvarez, J., D. Bicout, A. Boklund, A. Bøtner, K. Depner, S. J. More, H. Roberts, K. Stahl, H. Thulke, A. Viltrop and S. Antoniou, 2019: Research Gap Analysis on African Swine Fever. EFSA Journal, 17(8):5811,17, 5811.

Blázquez, E., J. Pujols, J. Segalés, C. Rodríguez, J. Ródenas, I. D. Kalmar, L. Heres and J. Polo, 2018: Effect of commercial spray-drying process on inactivation of African swine fever virus inoculated in concentrated porcine plasma .

Blázquez, E., J. Pujols, J. Segalés, F. Rodríguez, J. Crenshaw, C. Rodríguez, J. Ródenas and J. Polo, 2020: Commercial feed containing porcine plasma spiked with African swine fever virus is not infective in pigs when administered for 14 consecutive days. PLoS One, 15(7), e0235895. doi:10.1371/journal.pone.0235895.

Dee, S. A., F. V. Bauermann, M. C. Niederwerder, A. Singrey, T. Clement, M. de Lima, C. Long, G. Patterson, M. A. Sheahan, A. M. M. Stoian, V. Petrovan, C. K. Jones, J. De Jong, J. Ji, G. D. Spronk, L. Minion, J. Christopher-Hennings, J. J. Zimmerman, R. R. R. Rowland, E. Nelson, P. Sundberg and D. G. Diel, 2018: Survival of viral pathogens in animal feed ingredients under transboundary shipping models. PLoS One,13, e0194509.

Fischer, M., M. Mohnke, C. Probst, J. Pikalo, F. J. Conraths, M. Beer and S. Blome, 2020: Stability of African swine fever virus on heat-treated field crops. Transbound Emerg Dis .

Guinat, C., A. Gogin, S. Blome, G. Keil, R. Pollin, D. U. Pfeiffer and L. Dixon, 2016: Transmission routes of African swine fever virus to domestic pigs: current knowledge and future research directions. Vet Rec, 178, 262-267.

Heuschele, W. P., 1967: Studies on the pathogenesis of African swine fever. I. Quantitative studies on the sequential development of virus in pig tissues. Arch Gesamte Virusforsch, 21(3), 349-356. doi:10.1007/bf01241735.

Hoffmann, B., K. Depner, H. Schirrmeier and M. Beer, 2006: A universal heterologous internal control system for duplex real-time RT-PCR assays used in a detection system for pestiviruses. J Virol Methods,136, 200-209.

Kalmar, I. D., A. B. Cay and M. Tignon, 2018: Sensitivity of African swine fever virus (ASFV) to heat, alkalinity and peroxide treatment in presence or absence of porcine plasma. Vet Microbiol, 219, 144-149.

King, D. P., S. M. Reid, G. H. Hutchings, S. S. Grierson, P. J. Wilkinson, L. K. Dixon, A. D. Bastos and T. W. Drew, 2003: Development of a TaqMan PCR assay with internal amplification control for the detection of African swine fever virus. J Virol Methods, 107, 53-61.

Perez-Bosque, A., J. Polo and D. Torrallardona, 2016: Spray dried plasma as an alternative to antibiotics in piglet feeds, mode of action and biosafety. Porcine Health Manag, 2, 16.

Polo, J., E. Blázquez, J. Pujols, J. Segalés and C. Rodríguez, 2019:Inactivation of African Swine Fever virus inoculated in porcine fresh plasma by ultraviolet $(U V-C)$ light .

Polo, J., J. D. Quigley, L. E. Russell, J. M. Campbell, J. Pujols and P. D. Lukert, 2005: Efficacy of spraydrying to reduce infectivity of pseudorabies and porcine reproductive and respiratory syndrome (PRRS) viruses and seroconversion in pigs fed diets containing spray-dried animal plasma. J Anim Sci, 83, 19331938.

Pujols, J., R. Rosell, L. Russell, J. Campbell, J. Crenshaw, E. Weaver, C. Rodriguez, J. Rodenas and J. Polo, 2007: Inactivation of swine vesicular disease virus in porcine plasma by spray-drying. Proceedings of the 38th Annual Meeting of the American Association of Swine Veterinarians, Orlando, FL. , 281-283.

Pujols, J. and J. Segalés, 2014: Survivability of porcine epidemic diarrhea virus (PEDV) in bovine plasma submitted to spray drying processing and held at different time by temperature storage conditions. Vet Microbiol, 174, 427-432. 
Reed, L. J. and H. Muench, 1938: A simple method of estimating fifty per cent endpoints. Am J Epidemiol, 27, 493-497. doi:doi:10.1093/oxfordjournals.aje.a118408 \%J American Journal of Epidemiology.

\section{Figure Legends}

Figure 1: Copy numbers of virus suspension (VS) and inoculum (SDPP) at $4^{\circ} \mathrm{C}(\mathrm{A})$ and room temperature (B) over time. Open circles indicate individual replicate values, solid lines and error bars represent the mean and standard deviation. Neg: negative result.

Figure 2: Representation of the viral load as copy numbers (A) and of virus titers (B) after blind passage. Each open circle represents an individual replicate value, solid lines and error bars represent the mean and standard deviation. Neg: negative result.

\section{Supplement}

Table S1: Real-time PCR analysis of virus suspension and inoculum (SDPP)

Table S2: Real-time PCR analysis after blind passage

Table S3: Endpoint titers of virus suspension as $\mathrm{HAD}_{50} / \mathrm{ml}$
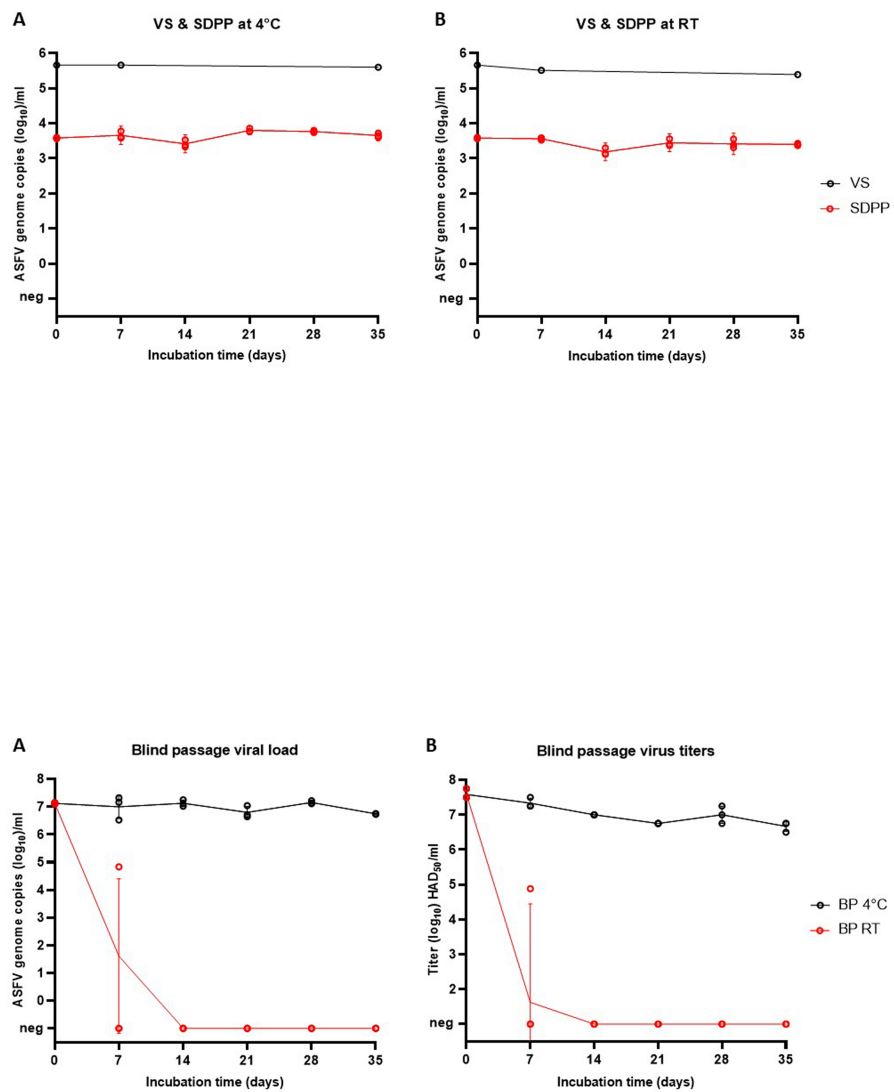